\title{
RESOLUTION OF DEGREE $\leq 6$ ALGEBRAIC EQUATIONS BY GENUS TWO THETA CONSTANTS
}

\section{ANGEL ZHIVKOV}

Communicated by Ivaïlo M. Mladenov

\begin{abstract}
We adjoin complete first kind Abelian integrals of genus two to resolve the general sextic equation $c_{0} z^{6}+c_{1} z^{5}+\cdots+c_{6}=0$ with simple zeros by genus two theta constants (Thetanullwerten). Using the same formulas, we also resolve each algebraic equation of degree five, four or three. It is shown that the monodromy group of a sextic is isomorphic to the second congruence sub-group $\Gamma(2)$ of the symplectic group $\mathrm{Sp}_{4}(\mathbb{Z})$.
\end{abstract}

\section{Introduction}

Diophantus was the first to expound ("Arithmetica", $3^{r d}$ century B.C.) the solution of the quadratic equation $a x^{2}+b x+c=0$. The solution of the cubic equation is nowadays known as Cardano formula and was derived in 1515 by del Ferro. In 1545 was published Ferrari's method for resolving by radicals of any quartic.

During the next three hundred years, fruitless efforts were made to find a solution by radicals of the quintic and higher degrees equations, which coefficients are letters. It was finally demonstrated by Abel [1] in 1826 that such solution does not exist. A complete answer to the question when an algebraic equation is solvable by radicals was given by Galois [6] about the year 1830 .

These discoveries of Abel and Galois had been followed by remarkable results of Hermite and Kronecker: in 1858 they proved that we can resolve any quintic extracting a cubic root, three square roots and using an elliptic modular function [9], [13]. That solution was in fact analogous to the formula

$$
\sqrt[n]{a}=\exp \left(\frac{1}{n} \int_{1}^{a} \frac{\mathrm{d} x}{x}\right)
$$

but the exponent replaced by an elliptic modular function and the integral $\int \frac{\mathrm{d} x}{x}$ by an elliptic integral. 
Kronecker thought that the resolution of the quintic would be a special case of a more general theorem which might exist. This hypothesis was realized in a few cases by Klein [12] and Jordan [11] showed that any algebraic equation is solvable by modular functions.

In 1984 Umemura [14] (see Appendix 1 in Mumford's book) realized Kronecker's idea, deducing from a formula of Thomae [17] a root of arbitrary polynomial by Siegel modular forms. This solution was expressed by genus $\left[\frac{n+2}{2}\right]$ theta constants related with the hyperelliptic curve

$$
R_{n}: \begin{cases}w^{2}=z(z-1) P_{n}(z) & \text { if } \mathrm{n} \text { is odd } \\ w^{2}=z(z-1)(z-2) P_{n}(z) & \text { if } \mathrm{n} \text { is even }\end{cases}
$$

where $P_{n}(z)$ is a $n^{t h}$ degree polynomial, whose roots are to be found. Especially $R_{6}$ is a genus four hyperelliptic curve and its period matrix depends on ten parameters which, however, are not free: there exists one Schottky relation between them and two Schottky-type relations extracting hyperelliptic between general genus four curves.

The aim of this paper is to resolve the equation $P_{6}(z)=0$ by genus two theta constants. In contrast to Umemura's solution in theta constants of genus four, our genus two theta functions are free of any restrictions. More precisely, any $(2 \times 2)$ Riemann matrix fits in the formulas. It seems that these formulas (Theorem 1) could not be simplified.

Similar formulae were derived by Guàrdia [8] without mentioning that all constants in Theorem 1 are free. Moreover, we resolve each algebraic equation of degree five, four or three, specifying correspondingly $\{\infty\},\{\infty, 0\}$ or $\{\infty, 0,1\}$ to be the roots of $P_{6}(z)$.

We give both easy and transparent proof that the expressions in Theorem 1 are the roots of $P_{6}(z)$ indeed and in particular, Thomae's formulas have been avoided. A simple idea will be used: if $\Delta$ denotes the Riemann theta divizor for genus two algebraic curve $R: w^{2}=P_{6}(z)$ and $\mathcal{A}$ denotes Abel's map, then the Riemann theta function $f(q):=\theta(\mathcal{A}(q-\Delta))$ vanishes identically for $q \in R$. Differentiating $f(q)$ and setting $q=(z, w)=\left(z_{j}, 0\right)$ gives explicitly the root $z_{j}$ of $P_{6}(z)$.

In Section 4 we study the monodromy group of the general sextic. This group turns out to be the second congruence sub-group $\Gamma(2)$ of the symplectic group $\mathrm{Sp}_{4}\left(\mathbb{F}_{2}\right)$. In Section 6 we discuss the theorem of Torelli in the case of genus two Riemann surfaces. 


\section{Explicit Roots of Degree Six Polynomials}

In this section we establish explicit expressions for the roots $z_{1}, z_{2}, \ldots, z_{6}$ of an arbitrary sextic

$$
P_{6}(z):=c_{0} z^{6}+c_{1} z^{5}+\cdots+c_{6}, \quad c_{j} \in \mathbb{C}, \quad c_{0} \neq 0
$$

by means of genus two theta constants.

The complex Sturm theorem [18] says that there exists an algorithm of separation the roots of $P_{6}(z)$. Further we shall consider each root $z_{j}$ to be simple and located inside some circle $U_{j} \in \mathbb{C}$.

Choose an arbitrary order $z_{1}, z_{2}, \ldots, z_{6}$ of the already localized roots and fix some paths $\gamma_{12}, \gamma_{34}$ and $\gamma_{56}$ to join correspondingly $U_{1}$ with $U_{2}, U_{3}$ with $U_{4}$ and $U_{5}$ with $U_{6}$, as in figure (1b) below. This defines two branches of the function

$$
w= \pm \sqrt{P_{6}(z)} \quad \text { for all } \quad z \in \mathbb{C}-U_{1}-\cdots-U_{6}-\gamma_{12}-\gamma_{34}-\gamma_{56}
$$

and when $z$ crosses some $\gamma_{i j}$, the sign of $w$ has to be chanced. More generally, $w$ is a well-defined meromorphic function on the genus two Riemann surface

$$
R: w^{2}=P_{6}(z)
$$

Let us fix a canonical basis of cycles $a_{1}, a_{2}, b_{1}, b_{2}$ on $R$ with intersection indexes

$$
a_{k} \circ a_{l}=b_{k} \circ b_{l}=0, \quad a_{k} \circ b_{l}=\delta_{k l}
$$

and such that the projection on the $z$-plane $z_{*} b_{1}$ surrounds $U_{1}$ and $U_{2}$, while $z_{*} a_{1}$ surrounds $U_{2}$ and $U_{3}, z_{*} a_{2}$ surrounds $U_{4}$ and $U_{5}, z_{*} b_{2}$ surrounds $U_{5}$ and $U_{6}$. Alternatively, chosen first the projections $z_{*} a_{k}$ and $z_{*} b_{k}$, then the cycles $a_{k} \subset$ $z^{-1}\left(z_{*} a_{k}\right)$ and $b_{k} \subset z^{-1}\left(z_{*} b_{k}\right)$.

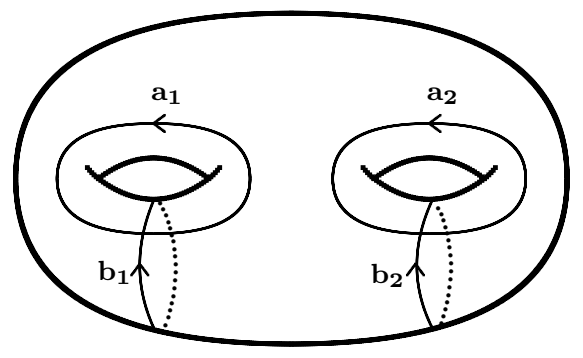

Figure 1a. A Riemann surface $R$. 


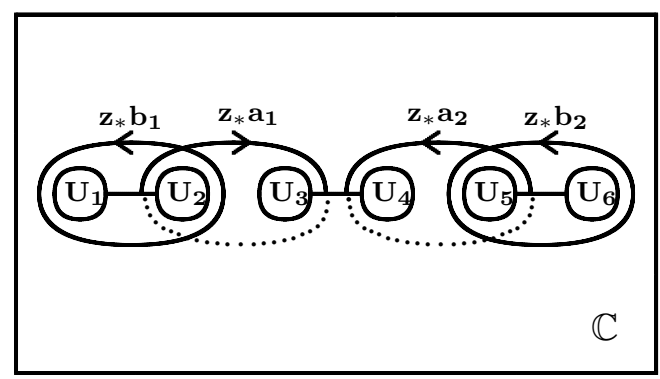

Figure 1b. Cycles in the $z$-plane.

Now we can define the following first kind complete Abelian integrals

$$
\begin{array}{llll}
\sigma_{11}=\oint_{a_{1}} \frac{\mathrm{d} z}{w}, & \sigma_{12}=\oint_{a_{2}} \frac{\mathrm{d} z}{w}, & \rho_{11}=\oint_{b_{1}} \frac{\mathrm{d} z}{w}, & \rho_{12}=\oint_{b_{2}} \frac{\mathrm{d} z}{w} \\
\sigma_{21}=\oint_{a_{1}} \frac{z \mathrm{~d} z}{w}, & \sigma_{22}=\oint_{a_{2}} \frac{z \mathrm{~d} z}{w}, & \rho_{21}=\oint_{b_{1}} \frac{z \mathrm{~d} z}{w}, & \rho_{22}=\oint_{b_{2}} \frac{z \mathrm{~d} z}{w} .
\end{array}
$$

It turns out that the numbers $\sigma_{11}, \sigma_{12}, \sigma_{21}, \sigma_{22}, \rho_{11}, \rho_{12}, \rho_{21}$ and $\rho_{22}$ contain all information we need and even arbitrary seven of them do.

Denote by $\sigma^{11}, \sigma^{12}, \sigma^{21}, \sigma^{22}$ the normalizing constants, i.e.,

$$
\left(\begin{array}{ll}
\sigma^{11} & \sigma^{12} \\
\sigma^{21} & \sigma^{22}
\end{array}\right)\left(\begin{array}{llll}
\sigma_{11} & \sigma_{12} & \rho_{11} & \rho_{12} \\
\sigma_{21} & \sigma_{22} & \rho_{21} & \rho_{22}
\end{array}\right)=\left(\begin{array}{llll}
1 & 0 & \Omega_{11} & \Omega_{12} \\
0 & 1 & \Omega_{21} & \Omega_{22}
\end{array}\right)
$$

and, therefore

$$
\oint_{a_{k}} \frac{\sigma^{s 1}+\sigma^{s 2} z}{w} \mathrm{~d} z=\delta_{k s}, \quad \oint_{b_{k}} \frac{\sigma^{s 1}+\sigma^{s 2} z}{w} \mathrm{~d} z=\Omega_{s k}, \quad k, s=1,2 .
$$

It is a standard fact [7] that the period matrix

$$
\Omega:=\left(\begin{array}{ll}
\Omega_{11} & \Omega_{12} \\
\Omega_{21} & \Omega_{22}
\end{array}\right)
$$

is a $(2 \times 2)$ symmetric matrix and $\operatorname{Im} \Omega>0$.

This enables to define the Riemann theta function with argument $u=\left(u_{1}, u_{2}\right) \in$ $\mathbb{C}^{2}$ and characteristics $\alpha=\left(\alpha_{1}, \alpha_{2}\right), \beta=\left(\beta_{1}, \beta_{2}\right) \in \mathbb{Q}^{2}$ by the Fourier expansion

$$
\theta\left[\begin{array}{c}
\alpha \\
\beta
\end{array}\right](u, \Omega):=\sum_{n \in \mathbb{Z}^{2}} \exp 2 \pi \mathrm{i}\left(\frac{1}{2}(n+\alpha) \Omega+u+\beta\right)(n+\alpha)^{t} .
$$


This classical function obeys the laws

$$
\begin{gathered}
\theta\left[\begin{array}{c}
\alpha \\
\beta
\end{array}\right](u+M+N \Omega, \Omega)=\theta\left[\begin{array}{c}
\alpha \\
\beta
\end{array}\right](u, \Omega) \\
\times \exp 2 \pi \mathrm{i}\left(-\frac{1}{2} N \Omega N^{t}-u N^{t}+\alpha M^{t}-\beta N^{t}\right) \\
\theta\left[\begin{array}{l}
\alpha \\
\beta
\end{array}\right](u, \Omega)=\theta\left(u+\left[\begin{array}{l}
\alpha \\
\beta
\end{array}\right], \Omega\right) \cdot \exp 2 \pi \mathrm{i}\left(\frac{1}{2} \alpha \Omega+u+\beta\right) \alpha^{t}
\end{gathered}
$$

for all $M, N \in \mathbb{Z}^{2}, u \in \mathbb{C}^{2}, \theta(u, \Omega):=\theta\left[\begin{array}{ll}0 & 0 \\ 0 & 0\end{array}\right](u, \Omega)$ and since every $u \in \mathbb{C}^{2}$ can be written uniquely by its characteristics $\alpha, \beta \in \mathbb{R}^{2}$ as

$$
u=\alpha+\beta \Omega:=\left[\begin{array}{l}
\alpha \\
\beta
\end{array}\right]=\left[\begin{array}{ll}
\alpha_{1} & \alpha_{2} \\
\beta_{1} & \beta_{2}
\end{array}\right]
$$

the use of the notation $\left[\begin{array}{l}\alpha \\ \beta\end{array}\right]$ for the points of $\mathbb{C}^{2}$ if not misleading.

When $\alpha$ and $\beta$ are half-integers, an and if $4 \alpha \beta^{t} \equiv 0 \bmod 2$, then the point $\left[\begin{array}{l}\alpha \\ \beta\end{array}\right] \in \mathbb{C}^{2}$ is called even half-period and $\theta\left[\begin{array}{l}\alpha \\ \beta\end{array}\right](u, \Omega)$ is an even function with regard to $u$, else if $4 \alpha \beta^{t} \equiv 1 \bmod 2$, the point $\left[\begin{array}{c}\alpha \\ \beta\end{array}\right]$ is called odd half-period and $\theta\left[\begin{array}{l}\alpha \\ \beta\end{array}\right](u, \Omega)$ is an odd function.

Recall also that the two-dimensional complex torus

$$
J(R):=\mathbb{C}^{2} /\left\{M+N \Omega ; M, N \in \mathbb{Z}^{2}\right\}
$$

is named Jacobian of $R$ and the Abel's map is defined by

$$
\begin{aligned}
\mathcal{A}: R & \rightarrow J(R) \\
q & \mapsto \mathcal{A}(q):=\left(\int_{q_{0}}^{q} \frac{\sigma^{11}+\sigma^{12} z}{w} \mathrm{~d} z, \int_{q_{0}}^{q} \frac{\sigma^{21}+\sigma^{22} z}{w} \mathrm{~d} z\right)
\end{aligned}
$$

where $q_{0}$ is an arbitrary but fixed point on $R$. An easy computation shows that

$$
\int_{z_{1}}^{z_{2}} \frac{\sigma^{s 1}+\sigma^{s 2} z}{w} \mathrm{~d} z=\frac{1}{2} \oint_{z_{*} b_{1}} \frac{\sigma^{s 1}+\sigma^{s 2} z}{w} \mathrm{~d} z=\frac{1}{2} \Omega_{s 1}, \quad s=1,2
$$

and hence if $q_{m}:=\left(z=z_{m}, w=0\right)$ denotes the $m^{t h}$ Weierstrass point on $R$,

$$
\mathcal{A}\left(q_{2}\right)-\mathcal{A}\left(q_{1}\right)=\left[\begin{array}{ll}
\frac{1}{2} & 0 \\
0 & 0
\end{array}\right] \quad \text { on } \quad J(R)
$$


Likewise, we have next identities on $J(R)$

$$
\begin{array}{ll}
\mathcal{A}\left(q_{3}\right)-\mathcal{A}\left(q_{2}\right)=\left[\begin{array}{ll}
0 & 0 \\
\frac{1}{2} & 0
\end{array}\right], & \mathcal{A}\left(q_{4}\right)-\mathcal{A}\left(q_{3}\right)=\left[\begin{array}{ll}
\frac{1}{2} & \frac{1}{2} \\
0 & 0
\end{array}\right] \\
\mathcal{A}\left(q_{5}\right)-\mathcal{A}\left(q_{4}\right)=\left[\begin{array}{ll}
0 & 0 \\
0 & \frac{1}{2}
\end{array}\right], & \mathcal{A}\left(q_{6}\right)-\mathcal{A}\left(q_{5}\right)=\left[\begin{array}{ll}
0 & \frac{1}{2} \\
0 & 0
\end{array}\right]
\end{array}
$$

which suggests to associate each Weierstrass points $q_{j}$ with an odd half-period as follows:

$$
\begin{aligned}
& q_{1} \leftrightarrow\left[\eta_{1}\right]:=\left[\begin{array}{cc}
\frac{1}{2} & \frac{1}{2} \\
0 & \frac{1}{2}
\end{array}\right], \quad q_{2} \leftrightarrow\left[\eta_{2}\right]:=\left[\begin{array}{ll}
0 & \frac{1}{2} \\
0 & \frac{1}{2}
\end{array}\right], \quad q_{3} \leftrightarrow\left[\eta_{3}\right]:=\left[\begin{array}{ll}
0 & \frac{1}{2} \\
\frac{1}{2} & \frac{1}{2}
\end{array}\right] \\
& q_{4} \leftrightarrow\left[\eta_{4}\right]:=\left[\begin{array}{cc}
\frac{1}{2} & 0 \\
\frac{1}{2} & \frac{1}{2}
\end{array}\right], \quad q_{5} \leftrightarrow\left[\eta_{5}\right]:=\left[\begin{array}{cc}
\frac{1}{2} & 0 \\
\frac{1}{2} & 0
\end{array}\right], \quad q_{6} \leftrightarrow\left[\eta_{6}\right]:=\left[\begin{array}{cc}
\frac{1}{2} & \frac{1}{2} \\
\frac{1}{2} & 0
\end{array}\right] .
\end{aligned}
$$

Consequently for all $m, s=1,2, \ldots, 6$,

$$
\mathcal{A}\left(q_{m}-q_{s}\right):=\mathcal{A}\left(q_{m}\right)-\mathcal{A}\left(q_{s}\right)=\left[\eta_{m}\right]-\left[\eta_{s}\right] \quad \text { on } \quad J(\Gamma) .
$$

To have more compact expressions for the roots of $P_{6}(z)$, we shall write

$$
\theta_{s}\left[\eta_{m}\right]:=\frac{\partial}{\partial u_{s}} \theta\left[\eta_{m}\right]\left(\left(u_{1}, u_{2}\right), \Omega\right)_{u_{1}=u_{2}=0}, \quad s=1,2
$$

for the first partial derivatives of the thetas, taken at $u=0$.

Theorem 1. The roots of the sextic $P_{6}(z)$ are given by

$$
z_{m}=\frac{\sigma_{22} \theta_{1}\left[\eta_{m}\right]-\sigma_{21} \theta_{2}\left[\eta_{m}\right]}{\sigma_{12} \theta_{1}\left[\eta_{m}\right]-\sigma_{11} \theta_{2}\left[\eta_{m}\right]}, \quad m=1,2, \ldots, 6 .
$$

Proof: According to Riemann's theorem on the theta divisor [7], either the section

$$
f(q):=\theta\left[\eta_{1}\right]\left(\mathcal{A}\left(q-q_{1}\right), \Omega\right), \quad q \in R
$$

vanishes identically, or it has exactly two zeros on $R$ (integrating the logarithmic derivative $\mathrm{d} \ln f(q)$ taken within the Riemann surface $R$ dissected along the cycles $a_{1}, a_{2}, b_{1}$ and $b_{2}$ verifies this assertion). But for any $m \leq 6$

$$
\begin{aligned}
f\left(q_{m}\right) & =\theta\left[\eta_{1}\right]\left(\mathcal{A}\left(q_{m}-q_{1}\right), \Omega\right) \\
& =\operatorname{const}_{1} \cdot \theta\left(\left[\eta_{m}\right], \Omega\right) \\
& =\text { const }_{2} \cdot \theta\left[\eta_{m}\right](0, \Omega) \\
& =0
\end{aligned}
$$


since $\theta\left[\eta_{m}\right](u, \Omega)$ is an odd function with regard to $u$ subject $\left[\eta_{m}\right]$ be an odd halfperiod. These six zeros can happen only if $f(q)$ vanishes identically.

A chain of implications finishes the proof: for each $m=1, \ldots, 6$

$$
\begin{aligned}
& f(q)=\theta\left[\eta_{1}\right]\left(\mathcal{A}\left(q-q_{1}\right), \Omega\right) \equiv 0 \\
\Rightarrow \quad & \frac{\mathrm{d}}{\mathrm{d} w(q)} f(q)_{\left.\right|_{q=q_{m}}}=0 \\
\Leftrightarrow \quad & \sum_{s=1}^{2} \theta_{s}\left[\eta_{1}\right]\left(\mathcal{A}\left(q_{m}-q_{1}\right), \Omega\right) \cdot \frac{\mathrm{d}}{\mathrm{d} w(q)} \int_{z\left(q_{1}\right)}^{z(q)} \frac{\sigma^{s 1}+\sigma^{s 2} z(q)}{w(q)} \mathrm{d} z(q)_{q_{m}=q_{m}}=0 \\
\Leftrightarrow \quad & \left.\sum_{s=1}^{2} \theta_{s}\left[\eta_{m}\right] \cdot \frac{\mathrm{d}}{\mathrm{d} w(q)} \int_{w\left(q_{1}\right)} \frac{\sigma^{s 1}+\sigma^{s 2} z(q)}{w(q)} \cdot \frac{2 w(q) \mathrm{d} w(q)}{P_{6}^{\prime}(z(q))}\right|_{q=q_{m}}=0 \\
\Leftrightarrow \quad & \sum_{s=1}^{2} \theta_{s}\left[\eta_{m}\right] \frac{\sigma^{s 1}+\sigma^{s 2} z\left(q_{m}\right)}{P_{6}^{\prime}\left(z\left(q_{m}\right)\right)}=\frac{1}{P_{6}^{\prime}\left(z_{m}\right)} \sum_{s=1}^{2} \theta_{s}\left[\eta_{m}\right] \cdot\left(\sigma^{s 1}+\sigma^{s 2} z_{m}\right)=0 \\
\Leftrightarrow \quad & z_{m}=-\frac{\sigma^{11} \theta_{1}\left[\eta_{m}\right]+\sigma^{21} \theta_{2}\left[\eta_{m}\right]}{\sigma^{12} \theta_{1}\left[\eta_{m}\right]+\sigma^{22} \theta_{2}\left[\eta_{m}\right]}=\frac{\sigma_{22} \theta_{1}\left[\eta_{m}\right]-\sigma_{21} \theta_{2}\left[\eta_{m}\right]}{\sigma_{12} \theta_{1}\left[\eta_{m}\right]-\sigma_{11} \theta_{2}\left[\eta_{m}\right]}
\end{aligned}
$$

and we have used $w^{2}=P_{6}(z)$ to deduce $2 w \mathrm{~d} w=P_{6}^{\prime}(z) \mathrm{d} z$.

\section{The Algorithm of Extracting the Roots}

In this short section we briefly recall the procedure for computing the roots of any fixed sextic $P_{6}(z)$ with complex coefficients and simple roots.

- Apply the complex Sturm theorem to localize the roots $z_{1}, z_{2}, \ldots, z_{6}$ of $P_{6}(z)$.

- Fix $z$-images $z_{*} a_{1}, z_{*} a_{2}, z_{*} b_{1}, z_{*} b_{2}$ of the cycles $a_{1}, a_{2}, b_{1}, b_{2}$ as in Fig. $1 \mathrm{~b}$.

For $w=\sqrt{P_{6}(z)}$, compute the integrals

$$
\begin{aligned}
& \sigma_{11}=\oint_{z_{*} a_{1}} \frac{\mathrm{d} z}{w}, \quad \sigma_{12}=\oint_{z_{*} a_{2}} \frac{\mathrm{d} z}{w}, \quad \rho_{11}=\oint_{z_{*} b_{1}} \frac{\mathrm{d} z}{w}, \quad \rho_{12}=\oint_{z_{*} b_{2}} \frac{\mathrm{d} z}{w} \\
& \sigma_{21}=\oint_{z_{*} a_{1}} \frac{z \mathrm{~d} z}{w}, \quad \sigma_{22}=\oint_{z_{*} a_{2}} \frac{z \mathrm{~d} z}{w}, \quad \rho_{21}=\oint_{z_{*} b_{1}} \frac{z \mathrm{~d} z}{w}, \quad \rho_{22}=\oint_{z_{*} b_{2}} \frac{z \mathrm{~d} z}{w} .
\end{aligned}
$$


- Compute the period matrix

$$
\Omega:=\left(\begin{array}{ll}
\sigma_{11} & \sigma_{12} \\
\sigma_{21} & \sigma_{22}
\end{array}\right)^{-1}\left(\begin{array}{ll}
\rho_{11} & \rho_{12} \\
\rho_{21} & \rho_{22}
\end{array}\right) .
$$

- Write down the roots

$$
z_{m}=\frac{\sigma_{22} \theta_{1}\left[\eta_{m}\right]-\sigma_{21} \theta_{2}\left[\eta_{m}\right]}{\sigma_{12} \theta_{1}\left[\eta_{m}\right]-\sigma_{11} \theta_{2}\left[\eta_{m}\right]}, \quad m=1,2, \ldots, 6
$$

where

$$
\begin{gathered}
{\left[\eta_{1}\right],\left[\eta_{2}\right], \ldots,\left[\eta_{6}\right]=\left[\begin{array}{ll}
\frac{1}{2} & \frac{1}{2} \\
0 & \frac{1}{2}
\end{array}\right],\left[\begin{array}{ll}
0 & \frac{1}{2} \\
0 & \frac{1}{2}
\end{array}\right],\left[\begin{array}{ll}
0 & \frac{1}{2} \\
\frac{1}{2} & \frac{1}{2}
\end{array}\right],\left[\begin{array}{cc}
\frac{1}{2} & 0 \\
\frac{1}{2} & \frac{1}{2}
\end{array}\right],\left[\begin{array}{cc}
\frac{1}{2} & 0 \\
\frac{1}{2} & 0
\end{array}\right],\left[\begin{array}{cc}
\frac{1}{2} & \frac{1}{2} \\
\frac{1}{2} & 0
\end{array}\right]} \\
\theta_{s}\left[\begin{array}{ll}
\alpha_{1} & \alpha_{2} \\
\beta_{1} & \beta_{2}
\end{array}\right]=-2 \pi \sum_{n_{1}, n_{2} \in \mathbb{Z}}(-1)^{2 \beta_{1} n_{1}+2 \beta_{2} n_{2}}\left(n_{s}+\alpha_{s}\right) \times \\
\times q_{11}^{\left(n_{1}+\alpha_{1}\right)^{2}} q_{12}^{2\left(n_{1}+\alpha_{1}\right)\left(n_{2}+\alpha_{2}\right)} q_{22}^{\left(n_{2}+\alpha_{2}\right)^{2}}
\end{gathered}
$$

and $q_{r s}:=\exp \left(\pi \mathrm{i} \Omega_{r s}\right)$ for $r, s=1,2$.

\section{Monodromy Group}

The algorithm of computing the roots of a polynomial $P_{6}(z)$ requires to fix their order $z_{1}, z_{2}, \ldots, z_{6}$. Intending to study arising monodromy, we introduce certain groups, normal sub-groups, factor-groups, exact sequences and homomorphisms

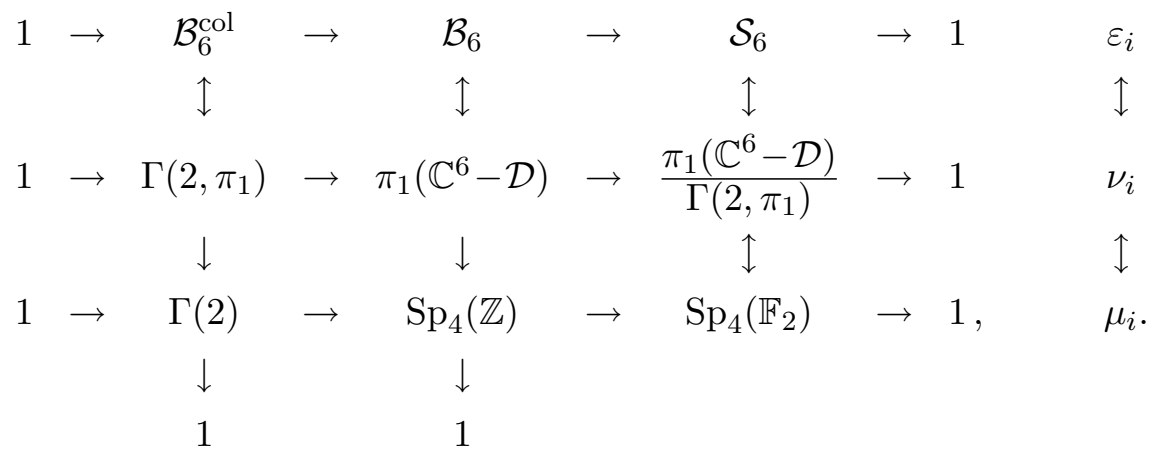

By $\mathcal{B}_{6}$ we have denoted the braid group [2], [5] with six (braid) strings, five generators $\varepsilon_{1}, \varepsilon_{2}, \ldots, \varepsilon_{5}$ and ten relations

$$
\begin{array}{ll}
\varepsilon_{s} \varepsilon_{s+1} \varepsilon_{s}=\varepsilon_{s+1} \varepsilon_{s} \varepsilon_{s+1} & \text { for } s=1,2,3,4 \\
\varepsilon_{i} \varepsilon_{s}=\varepsilon_{s} \varepsilon_{i} & \text { for } i-s>1, i, s=1, \ldots, 5 .
\end{array}
$$


Each braid can be considered as a continuous map

$$
\begin{aligned}
& f:[0,1] \rightarrow \mathbb{C}^{6}-\mathcal{D}:=\left\{\left(z_{1}, \ldots, z_{6}\right) \in \mathbb{C}^{6} ; z_{i} \neq z_{s} \text { for } i \neq s\right\} \\
& f(0)=f(1)
\end{aligned}
$$

modulo homotopy of $f$, which implies the braid group $\mathcal{B}_{6}$ coincides with the fundamental group $\pi_{1}=\pi_{1}\left(\mathbb{C}^{6}-\mathcal{D}\right)$ and the points $\left(z_{1}, \ldots, z_{6}\right)$ from the configuration space $\mathbb{C}^{6}-\mathcal{D}$ are the root tuples of $P_{6}(z)$. Denote by $\nu_{1}, \nu_{2}, \ldots, \nu_{5}$ the correspondent generators of $\pi_{1}\left(\mathbb{C}^{6}-\mathcal{D}\right)$.

Let $\mathcal{B}_{6}^{\text {col }}$ be the group of coloured braids with six strings, i.e., the normalizer of the sub-group of $\mathcal{B}_{6}$, generated by the squares $\varepsilon_{1}^{2}, \varepsilon_{2}^{2}, \ldots, \varepsilon_{5}^{2}$. Thus the factor-group $\mathcal{B}_{6} / \mathcal{B}_{6}^{\text {col }}$ has generators $\varepsilon_{1}, \varepsilon_{2}, \ldots, \varepsilon_{5}$ related by

$$
\begin{array}{ll}
\varepsilon_{s} \varepsilon_{s+1} \varepsilon_{s}=\varepsilon_{s+1} \varepsilon_{s} \varepsilon_{s+1} & \text { for } s=1,2,3,4 \\
\varepsilon_{i} \varepsilon_{s}=\varepsilon_{s} \varepsilon_{i} & \text { for } i-s>1, i, s=1, \ldots, 5 \\
\varepsilon_{i}^{2}=1 & \text { for } i=1, \ldots, 5 .
\end{array}
$$

Observe that the same relations (2) but each $\varepsilon_{s}$ replaced by the elementary transposition

$$
\left(\begin{array}{cccccc}
1 & \ldots & s & s+1 & \ldots & 6 \\
1 & \ldots & s+1 & s & \ldots & 6
\end{array}\right)
$$

define the symmetric group $\mathcal{S}_{6}$ [5]. This gives rise of a natural surjective homomorphism $\mathcal{B}_{6} \rightarrow \mathcal{S}_{6}$, of the isomorphism

$$
\mathcal{B}_{6} / \mathcal{B}_{6}^{\text {col }} \cong \mathcal{S}_{6}
$$

as well as the exact sequence $1 \rightarrow \mathcal{B}_{6}^{\text {col }} \rightarrow \mathcal{B}_{6} \rightarrow \mathcal{S}_{6} \rightarrow 1$.

Recall now that the symplectic group $\mathrm{Sp}_{4}(\mathbb{Z})$ consists of all $4 \times 4$ integer matrises $\mu:=\left(\begin{array}{ll}A & B \\ C & D\end{array}\right),(A, B, C$ and $D$ are $2 \times 2$ matrices $)$, which change every canonical basis of cycles $a_{1}, a_{2}, b_{1}, b_{2}$ by the canonical basis

$$
\left(\begin{array}{c}
\widehat{a} \\
\widehat{b}
\end{array}\right):=\left(\begin{array}{cc}
A & B \\
C & D
\end{array}\right)\left(\begin{array}{c}
a \\
b
\end{array}\right), \quad(\widehat{a}, \widehat{b}, a, b):=\left(\begin{array}{cccc}
\widehat{a}_{1} & \widehat{a}_{2} & a_{1} & a_{2} \\
\widehat{b}_{1} & \widehat{b}_{2} & b_{1} & b_{2}
\end{array}\right)
$$

preserving the intersection indexes. Algebraically

$$
\left(\begin{array}{cc}
A & B \\
C & D
\end{array}\right)^{t}\left(\begin{array}{rr}
0 & I \\
-I & 0
\end{array}\right)\left(\begin{array}{ll}
A & B \\
C & D
\end{array}\right)=\left(\begin{array}{rr}
0 & I \\
-I & 0
\end{array}\right)
$$

or, equivalently

$$
A^{t} D-C^{t} B=I, \quad A^{t} C=C^{t} A, \quad B^{t} D=D^{t} B .
$$


Next figures demonstrate the correspondence between the braid $\varepsilon_{1}$, the monodromy $\nu_{1}$ and the symplectic change $\mu_{1}$

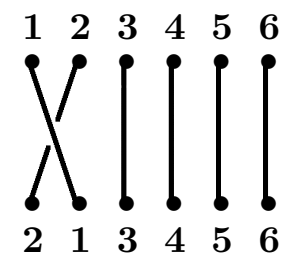

Figure 2a. Braid $\varepsilon_{1}$.

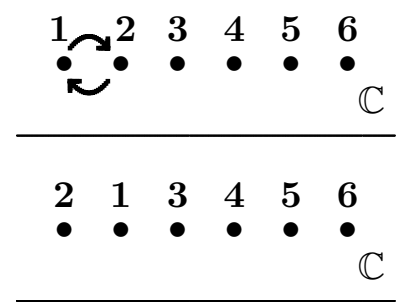

Figure 2b. Monodromy $\nu_{1}$.

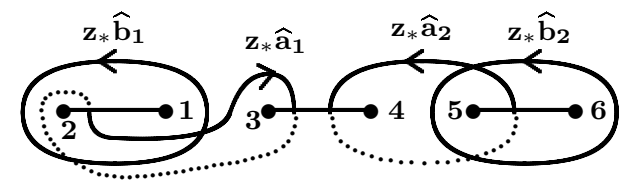

$\mathbb{C}$

Figure 2c. $\mu_{1}^{*}$-cycles in the $z$-plane.

After a straightforward computation of intersection indexes we calculate the matrix $\mu_{1} \in \mathrm{Sp}_{4}(\mathbb{Z})$ and, in the same way, $\mu_{2}, \mu_{3}, \mu_{4}, \mu_{5}$

$$
\begin{aligned}
\mu_{1} & =\left(\begin{array}{llll}
1 & 0 & 1 & 0 \\
0 & 1 & 0 & 0 \\
0 & 0 & 1 & 0 \\
0 & 0 & 0 & 1
\end{array}\right), \mu_{2}=\left(\begin{array}{rrrr}
1 & 0 & 0 & 0 \\
0 & 1 & 0 & 0 \\
-1 & 0 & 1 & 0 \\
0 & 0 & 0 & 1
\end{array}\right), \mu_{3}=\left(\begin{array}{llll}
1 & 0 & 1 & 1 \\
0 & 1 & 1 & 1 \\
0 & 0 & 1 & 0 \\
0 & 0 & 0 & 1
\end{array}\right) \\
\mu_{4} & =\left(\begin{array}{rrrr}
1 & 0 & 0 & 0 \\
0 & 1 & 0 & 0 \\
0 & 0 & 1 & 0 \\
0 & -1 & 0 & 1
\end{array}\right), \mu_{5}=\left(\begin{array}{llll}
1 & 0 & 0 & 0 \\
0 & 1 & 0 & 1 \\
0 & 0 & 1 & 0 \\
0 & 0 & 0 & 1
\end{array}\right) .
\end{aligned}
$$

One checks immediately that the relations (1) are fulfilled for $\mu_{s}$ as well which allows to conclude that the correspondence $\varepsilon_{s} \leftrightarrow \mu_{s}, s=1, \ldots, 5$, defines a homomorphism of groups $\mathcal{B}_{6} \rightarrow \mathrm{Sp}_{4}(\mathbb{Z})$. Moreover, this is a surjective homomorphism, as $\mu_{1}, \mu_{3}$,

$$
\mu_{0}=\left(\begin{array}{rrrr}
0 & 0 & 1 & 0 \\
0 & 0 & 0 & 1 \\
-1 & 0 & 0 & 0 \\
0 & -1 & 0 & 0
\end{array}\right), \mu_{6}=\left(\begin{array}{rrrr}
1 & 1 & 0 & 0 \\
0 & 1 & 0 & 0 \\
0 & 0 & 1 & 0 \\
0 & 0 & -1 & 1
\end{array}\right), \mu_{7}=\left(\begin{array}{rrrr}
0 & -1 & 0 & 0 \\
1 & 0 & 0 & 0 \\
0 & 0 & 0 & -1 \\
0 & 0 & 1 & 0
\end{array}\right)
$$


generate $\mathrm{Sp}_{4}(\mathbb{Z})$, see [3], and

$$
\begin{aligned}
\mu_{0} & :=\mu_{1} \mu_{2} \mu_{1} \mu_{4} \mu_{5} \mu_{4} \\
\mu_{6} & :=\mu_{1}^{-1} \mu_{3}^{-1} \mu_{5} \mu_{4} \mu_{3} \mu_{5}^{-1} \mu_{4}^{-1} \\
\mu_{7} & :=\left(\mu_{1} \mu_{2} \mu_{3} \mu_{4} \mu_{5}\right)^{3} \mu_{0} .
\end{aligned}
$$

On the other hand, the second congruence sub-group of $\operatorname{Sp}_{4}(\mathbb{Z})$

$$
\Gamma(2):=\left\{\mu \in \operatorname{Sp}_{4}(\mathbb{Z}) ; \mu \equiv I_{4 \times 4} \bmod 2\right\}
$$

can be defined as follows: first $\mu_{1}^{2}, \ldots, \mu_{5}^{2}$ generate some sub-group $\Gamma \subset \Gamma(2)$, then one normalizes $\Gamma$ to obtain $\Gamma(2)$ spanned by [14]

$$
\mu_{1}^{2}, \ldots, \mu_{5}^{2}, \mu_{6}^{2}=\mu_{1}^{-2} \mu_{5} \mu_{4} \mu_{3}^{2} \mu_{5}^{-2} \mu_{4}^{-1} \mu_{5}^{-1} \text { and } \mu_{7} \mu_{6}^{2} \mu_{7}^{-1}
$$

The factor-group $\operatorname{Sp}_{4}(\mathbb{Z}) / \Gamma(2)$ will be identified as $\operatorname{Sp}_{4}\left(\mathbb{F}_{2}\right), \mathbb{F}_{2}$ being the field with two elements.

Proposition 2. ([15]) The factor-group $\mathrm{Sp}_{4}\left(\mathbb{F}_{2}\right)$ and the symmetric group $\mathcal{S}_{6}$ are isomorphic via the correspondence $\varepsilon_{s} \leftrightarrow \mu_{s}, s=1, \ldots, 5$.

Proof: As we have a surjective homomorphism $\mathcal{B}_{6} \rightarrow \mathrm{Sp}_{4}(\mathbb{Z})$ and the correspondent sub-groups $\mathcal{B}_{6}^{\text {col }}$ and $\Gamma(2)$ are generated in an identical manner, the homomorphism $\mathcal{S}_{6} \rightarrow \mathrm{Sp}_{4}\left(\mathbb{F}_{2}\right)$ is surjective, too.

To establish an isomorphism, we have still to verify that the group $\operatorname{Sp}_{4}\left(\mathbb{F}_{2}\right)$ has order 720 , like $\mathcal{S}_{6}$ does. Indeed, consider a chain of sub-groups

$$
\begin{gathered}
\operatorname{Sp}_{4}\left(\mathbb{F}_{2}\right) \supset G_{1} \supset G_{2} \supset G_{3} \\
G_{1}=\left(\begin{array}{llll}
1 & * & * & * \\
0 & * & * & * \\
0 & * & * & * \\
0 & * & * & *
\end{array}\right), \quad G_{2}=\left(\begin{array}{cccc}
1 & 0 & 0 & 0 \\
0 & * & * & * \\
0 & * & * & * \\
0 & * & * & *
\end{array}\right), \quad G_{3}=\left(\begin{array}{llll}
1 & 0 & 0 & 0 \\
0 & 1 & 0 & 0 \\
0 & 0 & 1 & 0 \\
0 & * & 0 & 1
\end{array}\right)
\end{gathered}
$$

(the stars $*$ stand for 0 or 1 ). Given an arbitrary symplectic matrix $\mu$, there always exist suitable left and right multiplications by symplectic matrices to include the products successively in $G_{1}, G_{2}$ and $G_{3}$.

The index of $G_{1}$ in $\operatorname{Sp}_{4}\left(\mathbb{F}_{2}\right)$ equals 15 , since the first column of $\mu$ is not equal to $(0,0,0,0)$. The index $\left[G_{1}: G_{2}\right]=8$. The index $\left[G_{2}: G_{3}\right]=3$ as the second row could be $(0,1,0,0),(0,1,0,1)$ or $(0,0,0,1)$. The group $G_{3}$ has order two. Summing up, the order of $\operatorname{Sp}_{4}\left(\mathbb{F}_{2}\right)$ equals 15.8.3.2=720. 
Remark also next correspondence

$$
\begin{array}{cll}
\mathrm{Sp}_{4}(\mathbb{Z}) & -a_{1}, a_{2}, b_{2}, b_{2} \text { are not fixed } & -z_{1}, z_{2}, \ldots, z_{6} \text { are not fixed } \\
G_{1} & -b_{1} \text { is fixed } & -\left\{z_{1}, z_{2}\right\} \text { are fixed } \\
G_{2} & -b_{1}, a_{1} \text { are fixed } & -z_{1}, z_{2}, z_{3} \text { are fixed } \\
G_{3} & -b_{1}, a_{1}, a_{2} \text { are fixed } & -z_{1}, z_{2}, z_{3}, z_{6} \text { are fixed } \\
I & -b_{1}, a_{1}, a_{2}, b_{2} \text { are fixed } & -z_{1}, \ldots, z_{6} \text { are fixed } .
\end{array}
$$

Now we may formulate:

Theorem 3. The monodromy group of the family of genus two algebraic curves $w^{2}=P_{6}(z)$ is the second congruence sub-group $\Gamma(2) \subset \operatorname{Sp}_{4}(\mathbb{Z})$. The extended monodromy group for this family coincides with the Siegel modular group $\operatorname{Sp}_{4}(\mathbb{Z})$. More precisely, $\Gamma(2)$ leaves the roots $\left(z_{1}, \ldots, z_{6}\right)$ of $P_{6}(z)$ invariant, while the factor-group

$$
\operatorname{Sp}_{4}\left(\mathbb{F}_{2}\right)=\operatorname{Sp}_{4}(\mathbb{Z}) / \Gamma(2) \cong \mathcal{S}_{6}
$$

permutes effectively and transitively these roots.

There also exists an exact commutative diagram

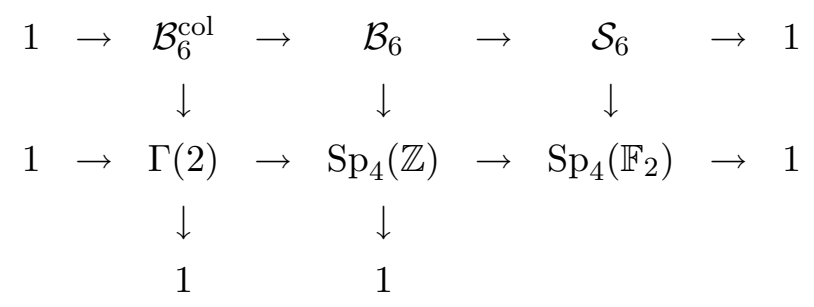

which relates the monodromy to the braid groups $\mathcal{B}_{6}$ and $\mathcal{B}_{6}^{\mathrm{col}}$.

To complete the point, let us take a symplectic matrix $\mu:=\left(\begin{array}{cc}A & B \\ C & D\end{array}\right) \in \operatorname{Sp}_{4}(\mathbb{Z})$ and give a hat $\widehat{\wedge}$ for every new object arising after the change $\left(\begin{array}{l}\widehat{a} \\ \widehat{b}\end{array}\right)=\mu \cdot\left(\begin{array}{l}a \\ b\end{array}\right)$ of the homology basis on $R$. Then [10]

$$
\begin{aligned}
& \widehat{\sigma}=\sigma A^{t}+\rho B^{t}, \quad \widehat{\rho}=\sigma C^{t}+\rho D^{t}, \quad \widehat{\Omega}=(C+D \Omega)(A+B \Omega)^{-1} \\
& \widehat{\mathcal{A}}(q)=\mathcal{A}(q) \cdot(A+B \Omega)^{-1} \\
& \widehat{u}=\left(\widehat{u}_{1}, \widehat{u}_{2}\right):=\left(u_{1}, u_{2}\right) \cdot(A+B \Omega)^{-1}=u \cdot(A+B \Omega)^{-1} \\
& {[\widehat{\eta}]=\left[\begin{array}{c}
\widehat{\alpha} \\
\widehat{\beta}
\end{array}\right]:=\left[\begin{array}{c}
\alpha A^{t}-\beta B^{t} \\
-\alpha C^{t}+\beta D^{t}
\end{array}\right]+\frac{1}{2}\left[\begin{array}{ll}
\left(A B^{t}\right)_{11} & \left(A B^{t}\right)_{22} \\
\left(C D^{t}\right)_{11} & \left(C D^{t}\right)_{22}
\end{array}\right]}
\end{aligned}
$$




$$
\begin{aligned}
& \widehat{\theta}[\widehat{\eta}](\widehat{u}, \widehat{\Omega})=\kappa \cdot \sqrt{\operatorname{det}(A+B \Omega)} \cdot \exp \left(\pi \mathrm{i} \widehat{u} B u^{t}\right) \cdot \theta[\eta](u, \Omega) \\
& \left(\widehat{\theta}_{1}[\widehat{\eta}], \widehat{\theta}_{2}[\widehat{\eta}]\right)=\kappa \cdot \sqrt{\operatorname{det}(A+B \Omega)} \cdot\left(\theta_{1}[\eta], \theta_{2}[\eta]\right) \cdot\left(A^{t}+\Omega B^{t}\right)
\end{aligned}
$$

where $\kappa$ is certain eight root of unity, independent on $u$ and $\Omega$. The last relation between theta-function-gradients yields for each $m=1,2, \ldots, 6$

$$
\left(\widehat{\theta}_{1}\left[\widehat{\eta}_{m}\right], \widehat{\theta}_{2}\left[\widehat{\eta}_{m}\right]\right) \cdot \widehat{\sigma}^{-1}=\kappa \cdot \sqrt{\operatorname{det}(A+B \Omega)} \cdot\left(\theta_{1}\left[\eta_{\widehat{m}}\right], \theta_{2}\left[\eta_{\widehat{m}}\right]\right) \cdot \sigma^{-1}
$$

and, henceforth, the invariant equality

$$
\widehat{z}_{m}=-\frac{\widehat{\sigma}^{11} \widehat{\theta}_{1}\left[\widehat{\eta}_{m}\right]+\widehat{\sigma}^{21} \widehat{\theta}_{2}\left[\widehat{\eta}_{m}\right]}{\widehat{\sigma}^{12} \widehat{\theta}_{1}\left[\widehat{\eta}_{m}\right]+\widehat{\sigma}^{22} \widehat{\theta}_{2}\left[\widehat{\eta}_{m}\right]}=-\frac{\sigma^{11} \theta_{1}\left[\eta_{\widehat{m}}\right]+\sigma^{21} \theta_{2}\left[\eta_{\widehat{m}}\right]}{\sigma^{12} \theta_{1}\left[\eta_{\widehat{m}}\right]+\sigma^{22} \theta_{2}\left[\eta_{\widehat{m}}\right]}=z_{\widehat{m}}
$$

where $\widehat{m}:=\left(\varepsilon_{i_{1}} \varepsilon_{i_{2}} \ldots \varepsilon_{i_{n}}\right)(m)$ subject $\mu=\mu_{i_{1}} \mu_{i_{2}} \ldots \mu_{i_{n}}$.

\section{A Resolution of Degree $<6$ Algebraic Equations}

1. All derived formulas about the roots of a sextic remain true for each quintic

$$
P_{5}(z)=c_{1} z^{5}+c_{2} z^{4}+\cdots+c_{6}
$$

with simple roots and $c_{1} \neq 0$. Letting $c_{0}=0$ implies a root, say $z_{6}=\infty$ and then the denominator $\sigma_{12} \theta_{1}\left[\eta_{6}\right]-\sigma_{11} \theta_{2}\left[\eta_{6}\right]$ vanishes, which defines both integrals $\sigma_{11}, \sigma_{12}$ up to a multiplicative constant $\xi$.

According to the classical Rosenhain formula [16], for $1 \leq m<s \leq 6$

$$
\theta_{1}\left[\eta_{m}\right] \theta_{2}\left[\eta_{s}\right]-\theta_{2}\left[\eta_{m}\right] \theta_{1}\left[\eta_{s}\right]=\pi^{2} \theta\left[e_{1}^{m, s}\right] \theta\left[e_{2}^{m, s}\right] \theta\left[e_{3}^{m, s}\right] \theta\left[e_{4}^{m, s}\right]
$$

where $\left[e_{1}^{m, s}\right], \ldots,\left[e_{4}^{m, s}\right]$ are the even half-periods for which $\left[e_{i}^{m, s}\right]+\left[\eta_{m}\right]+\left[\eta_{s}\right]$ is an odd half-period, $\theta[e]:=\theta[e](0, \Omega)$. Using $z_{1}+\cdots+z_{5}=-\frac{c_{2}}{c_{1}}$ to evaluate the constant $\xi$, we compute explicitly the roots of $P_{5}(z)$

$$
\begin{gathered}
z_{1}=\frac{\sigma_{22} \theta_{1}\left[\begin{array}{ll}
\frac{1}{2} & \frac{1}{2} \\
0 & \frac{1}{2}
\end{array}\right]-\sigma_{21} \theta_{2}\left[\begin{array}{ll}
\frac{1}{2} & \frac{1}{2} \\
0 & \frac{1}{2}
\end{array}\right]}{\xi \theta\left[\begin{array}{ll}
\frac{1}{2} & 0 \\
0 & \frac{1}{2}
\end{array}\right] \theta\left[\begin{array}{ll}
\frac{1}{2} & 0 \\
0 & 0
\end{array}\right] \theta\left[\begin{array}{ll}
0 & \frac{1}{2} \\
\frac{1}{2} & 0
\end{array}\right] \theta\left[\begin{array}{ll}
0 & \frac{1}{2} \\
0 & 0
\end{array}\right]}, \quad z_{2}=\frac{\sigma_{22} \theta_{1}\left[\begin{array}{ll}
0 & \frac{1}{2} \\
0 & \frac{1}{2}
\end{array}\right]-\sigma_{21} \theta_{2}\left[\begin{array}{ll}
0 & \frac{1}{2} \\
0 & \frac{1}{2}
\end{array}\right]}{\xi \theta\left[\begin{array}{ll}
0 & 0 \\
0 & \frac{1}{2}
\end{array}\right] \theta\left[\begin{array}{ll}
0 & 0 \\
0 & 0
\end{array}\right] \theta\left[\begin{array}{ll}
\frac{1}{2} & \frac{1}{2} \\
0 & 0
\end{array}\right] \theta\left[\begin{array}{ll}
0 & \frac{1}{2} \\
\frac{1}{2} & 0
\end{array}\right]} \\
z_{3}=\frac{\sigma_{22} \theta_{1}\left[\begin{array}{ll}
0 & \frac{1}{2} \\
\frac{1}{2} & \frac{1}{2}
\end{array}\right]-\sigma_{21} \theta_{2}\left[\begin{array}{ll}
0 & \frac{1}{2} \\
\frac{1}{2} & \frac{1}{2}
\end{array}\right]}{\xi \theta\left[\begin{array}{ll}
0 & 0 \\
\frac{1}{2} & \frac{1}{2}
\end{array}\right] \theta\left[\begin{array}{ll}
0 & 0 \\
\frac{1}{2} & 0
\end{array}\right] \theta\left[\begin{array}{ll}
\frac{1}{2} & \frac{1}{2} \\
0 & 0
\end{array}\right] \theta\left[\begin{array}{ll}
0 & \frac{1}{2} \\
0 & 0
\end{array}\right]}, \quad z_{4}=\frac{\sigma_{22} \theta_{1}\left[\begin{array}{ll}
\frac{1}{2} & 0 \\
\frac{1}{2} & \frac{1}{2}
\end{array}\right]-\sigma_{21} \theta_{2}\left[\begin{array}{ll}
\frac{1}{2} & 0 \\
\frac{1}{2} & \frac{1}{2}
\end{array}\right]}{\xi \theta\left[\begin{array}{ll}
\frac{1}{2} & \frac{1}{2} \\
\frac{1}{2} & \frac{1}{2}
\end{array}\right] \theta\left[\begin{array}{ll}
0 & 0 \\
0 & 0
\end{array}\right] \theta\left[\begin{array}{ll}
0 & 0 \\
\frac{1}{2} & 0
\end{array}\right] \theta\left[\begin{array}{ll}
\frac{1}{2} & 0 \\
0 & 0
\end{array}\right]}
\end{gathered}
$$




$$
\begin{gathered}
z_{5}=\frac{\sigma_{22} \theta_{1}\left[\begin{array}{ll}
\frac{1}{2} & 0 \\
\frac{1}{2} & 0
\end{array}\right]-\sigma_{21} \theta_{2}\left[\begin{array}{ll}
\frac{1}{2} & 0 \\
\frac{1}{2} & 0
\end{array}\right]}{\xi \theta\left[\begin{array}{ll}
\frac{1}{2} & \frac{1}{2} \\
\frac{1}{2} & \frac{1}{2}
\end{array}\right] \theta\left[\begin{array}{ll}
0 & 0 \\
0 & \frac{1}{2}
\end{array}\right] \theta\left[\begin{array}{ll}
0 & 0 \\
\frac{1}{2} & \frac{1}{2}
\end{array}\right] \theta\left[\begin{array}{ll}
\frac{1}{2} & 0 \\
0 & \frac{1}{2}
\end{array}\right]} \\
\xi:=-\frac{c_{1}}{c_{2}} \sum_{m=1}^{5} \frac{\sigma_{22} \theta_{1}\left[\eta_{m}\right]-\sigma_{21} \theta_{2}\left[\eta_{m}\right]}{\theta\left[e_{1}^{m, 6}\right] \theta\left[e_{2}^{m, 6}\right] \theta\left[e_{3}^{m, 6}\right] \theta\left[e_{4}^{m, 6}\right]} .
\end{gathered}
$$

In the case $c_{2}=0$ we define $\xi$ with the help of another formula of Viète.

2. Similar arguments hold for the polynomials

$$
P_{4}(z)=c_{1} z^{4}+c_{2} z^{3}+\cdots+c_{5}
$$

with $c_{1} c_{5} \neq 0, c_{6}=0$ and simple roots. In addition to $z_{6}=\infty$, we shall consider $z_{5}=0$, that is the above $z_{5}$-numerator vanishes. Hence

$$
\sigma_{21}: \sigma_{22}=\theta_{1}\left[\eta_{5}\right]: \theta_{2}\left[\eta_{5}\right]
$$

to conclude (using again Rosenhain's formula) the quartic $P_{4}(z)$ has roots

$$
\begin{array}{ccc}
z_{1}=\zeta \cdot \frac{\theta\left[\begin{array}{ll}
0 & 0 \\
\frac{1}{2} & 0
\end{array}\right] \theta\left[\begin{array}{ll}
\frac{1}{2} & \frac{1}{2} \\
0 & 0
\end{array}\right] \theta\left[\begin{array}{ll}
0 & 0 \\
0 & 0
\end{array}\right]}{\theta\left[\begin{array}{ll}
0 & \frac{1}{2} \\
\frac{1}{2} & 0
\end{array}\right] \theta\left[\begin{array}{ll}
\frac{1}{2} & 0 \\
0 & 0
\end{array}\right] \theta\left[\begin{array}{ll}
0 & \frac{1}{2} \\
0 & 0
\end{array}\right]}, & z_{2}=\zeta \cdot \frac{\theta\left[\begin{array}{ll}
0 & 0 \\
\frac{1}{2} & 0
\end{array}\right] \theta\left[\begin{array}{ll}
\frac{1}{2} & 0 \\
0 & 0
\end{array}\right] \theta\left[\begin{array}{ll}
0 & \frac{1}{2} \\
0 & 0
\end{array}\right]}{\theta\left[\begin{array}{ll}
0 & \frac{1}{2} \\
\frac{1}{2} & 0
\end{array}\right] \theta\left[\begin{array}{ll}
\frac{1}{2} & \frac{1}{2} \\
0 & 0
\end{array}\right] \theta\left[\begin{array}{ll}
0 & 0 \\
0 & 0
\end{array}\right]} \\
z_{3}=\zeta \cdot \frac{\theta\left[\begin{array}{ll}
0 & \frac{1}{2} \\
\frac{1}{2} & 0
\end{array}\right] \theta\left[\begin{array}{ll}
\frac{1}{2} & 0 \\
0 & 0
\end{array}\right] \theta\left[\begin{array}{ll}
0 & 0 \\
0 & 0
\end{array}\right]}{\theta\left[\begin{array}{ll}
0 & 0 \\
\frac{1}{2} & 0
\end{array}\right] \theta\left[\begin{array}{ll}
\frac{1}{2} & \frac{1}{2} \\
0 & 0
\end{array}\right] \theta\left[\begin{array}{ll}
0 & \frac{1}{2} \\
0 & 0
\end{array}\right]}, & z_{4}=\zeta \cdot \frac{\theta\left[\begin{array}{ll}
0 & \frac{1}{2} \\
\frac{1}{2} & 0
\end{array}\right] \theta\left[\begin{array}{ll}
\frac{1}{2} & \frac{1}{2} \\
0 & 0
\end{array}\right] \theta\left[\begin{array}{ll}
0 & \frac{1}{2} \\
0 & 0
\end{array}\right]}{\theta\left[\begin{array}{ll}
0 & 0 \\
\frac{1}{2} & 0
\end{array}\right] \theta\left[\begin{array}{ll}
\frac{1}{2} & 0 \\
0 & 0
\end{array}\right] \theta\left[\begin{array}{ll}
0 & 0 \\
0 & 0
\end{array}\right]}
\end{array}
$$

where $z_{1}+z_{2}+z_{3}+z_{4}=-\frac{c_{2}}{c_{1}}$ (or another formula of Viète if $c_{2}=0$ ) defines unambiguously the constant $\zeta$.

Multiplying by the least common denominator of $z_{1}, z_{2}, z_{3}, z_{4}$ simplifies the above expressions

$$
\begin{array}{ll}
z_{1}=\zeta_{1} \cdot \theta\left[\begin{array}{ll}
0 & 0 \\
\frac{1}{2} & 0
\end{array}\right]^{2} \theta\left[\begin{array}{cc}
\frac{1}{2} & \frac{1}{2} \\
0 & 0
\end{array}\right]^{2} \theta\left[\begin{array}{ll}
0 & 0 \\
0 & 0
\end{array}\right]^{2}, & z_{2}=\zeta_{1} \cdot \theta\left[\begin{array}{ll}
0 & 0 \\
\frac{1}{2} & 0
\end{array}\right]^{2} \theta\left[\begin{array}{cc}
\frac{1}{2} & 0 \\
0 & 0
\end{array}\right]^{2} \theta\left[\begin{array}{ll}
0 & \frac{1}{2} \\
0 & 0
\end{array}\right]^{2} \\
z_{3}=\zeta_{1} \cdot \theta\left[\begin{array}{ll}
0 & \frac{1}{2} \\
\frac{1}{2} & 0
\end{array}\right]^{2} \theta\left[\begin{array}{cc}
\frac{1}{2} & 0 \\
0 & 0
\end{array}\right]^{2} \theta\left[\begin{array}{ll}
0 & 0 \\
0 & 0
\end{array}\right]^{2}, & z_{4}=\zeta_{1} \cdot \theta\left[\begin{array}{ll}
0 & \frac{1}{2} \\
\frac{1}{2} & 0
\end{array}\right]^{2} \theta\left[\begin{array}{cc}
\frac{1}{2} & \frac{1}{2} \\
0 & 0
\end{array}\right]^{2} \theta\left[\begin{array}{ll}
0 & \frac{1}{2} \\
0 & 0
\end{array}\right]^{2}
\end{array}
$$

with a constant $\zeta_{1}$ specified like $\zeta$ did. 
3. In order to resolve the cubic equation

$$
P_{3}(z)=c_{2} z^{3}+c_{3} z^{2}+c_{4} z+c_{5}=0
$$

$\left(c_{2} c_{5} \neq 0\right.$, the roots $z_{1}, z_{2}, z_{3}$ are simple and $\neq 1$ ) via two-dimensional theta constants, we regard the quartic $P_{4}(z):=(z-1) P_{3}(z)$, namely suppose the above constant $\zeta_{1}$ specified upon $z_{4}=1$, whence

$$
z_{1}=\frac{\theta\left[\begin{array}{ll}
0 & 0 \\
\frac{1}{2} & 0
\end{array}\right]^{2} \theta\left[\begin{array}{ll}
0 & 0 \\
0 & 0
\end{array}\right]^{2}}{\theta\left[\begin{array}{ll}
0 & \frac{1}{2} \\
\frac{1}{2} & 0
\end{array}\right]^{2} \theta\left[\begin{array}{ll}
0 & \frac{1}{2} \\
0 & 0
\end{array}\right]^{2}}, \quad z_{2}=\frac{\theta\left[\begin{array}{ll}
0 & 0 \\
\frac{1}{2} & 0
\end{array}\right]^{2} \theta\left[\begin{array}{ll}
\frac{1}{2} & 0 \\
0 & 0
\end{array}\right]^{2}}{\theta\left[\begin{array}{ll}
0 & \frac{1}{2} \\
\frac{1}{2} & 0
\end{array}\right]^{2} \theta\left[\begin{array}{ll}
\frac{1}{2} & \frac{1}{2} \\
0 & 0
\end{array}\right]^{2}}, \quad z_{3}=\frac{\theta\left[\begin{array}{ll}
\frac{1}{2} & 0 \\
0 & 0
\end{array}\right]^{2} \theta\left[\begin{array}{ll}
0 & 0 \\
0 & 0
\end{array}\right]^{2}}{\theta\left[\begin{array}{cc}
\frac{1}{2} & \frac{1}{2} \\
0 & 0
\end{array}\right]^{2} \theta\left[\begin{array}{ll}
0 & \frac{1}{2} \\
0 & 0
\end{array}\right]^{2}} .
$$

Let us remark that these exact squares coinside with the roots of the cubic $P_{3}(z)$ due to Umemura [14], compare also with [4].

\section{Torelli Theorem for Genus Two Curves}

Every genus two Riemann surface $R$ is hyperelliptic and there always exist two meromorphic functions $w, z: R \rightarrow \mathbb{C P}^{1}$ such that $w^{2}=P_{6}(z)$ for certain sextic $P_{6}(z)$ with simple roots [7]. In accordance with our construction, fix an order of these roots and compute the period matrix $\Omega=\Omega(R)$ to define a rank four lattice

$$
\Lambda(\Omega):=\left\{M+N \Omega ; M, N \in \mathbb{Z}^{2}\right\} \subset \mathbb{C}^{2} .
$$

Then the classical Torelli theorem [7] claims that the Riemann surface $R$ can be restored by its Jacobian $J(R)=\mathbb{C}^{2} / \Lambda(\Omega)$, or, what is the same, by $\Lambda(\Omega)$.

On the other side, each $(2 \times 2)$ symmetric matrix $\Omega$ with $\operatorname{Im} \Omega>0$ defines a two-dimensional complex torus $T:=\mathbb{C}^{2} / \Lambda(\Omega)$ and then the Riemann surface

$$
R(\Omega): w^{2}=\prod_{m=1}^{6}\left(z-\frac{\theta_{1}\left[\eta_{m}\right]}{\theta_{2}\left[\eta_{m}\right]}\right)
$$

has a Jacobian $J(R)=T$ [4]. This formula effectively solves Torelli's theorem for genus two Riemann surfaces.

Notice that the symplectic group $\operatorname{Sp}_{4}(\mathbb{Z})$ preserves $\Lambda(\Omega)$ and thus the Riemann surface $R(\Omega)$ invariant, while the group-action

$$
z \mapsto \frac{h_{11} z+h_{12}}{h_{21} z+h_{22}}, \quad h:=\left(\begin{array}{ll}
h_{11} & h_{12} \\
h_{21} & h_{22}
\end{array}\right) \in \operatorname{PGL}_{2}(\mathbb{C}) \cong \operatorname{Aut}\left(\mathbb{C P}^{1}\right)
$$


leaves $R(\Omega)$ invariant in the sense that any Riemann surface

$$
R_{h}(\Omega): w^{2}=\prod_{m=1}^{6}\left(\frac{h_{11} z+h_{12}}{h_{21} z+h_{22}}-\frac{\theta_{1}\left[\eta_{m}\right]}{\theta_{2}\left[\eta_{m}\right]}\right)
$$

remains algebraically isomorphic to $R(\Omega)$.

Conversely, if $\Omega$ and $\Omega^{\prime}$ are two period matrices such that the lattices $\Lambda(\Omega)$ and $\Lambda\left(\Omega^{\prime}\right)$ are different, then they define by (4) two algebraically non-isomorphic Riemann surfaces $R(\Omega)$ and $R\left(\Omega^{\prime}\right)$. The variety of moduli of all algebraically non-isomorphic genus two Riemann surfaces can be written as

$$
\begin{aligned}
\mathcal{M}_{2} & =\{2 \times 2 \text { symmetric matrix } \Omega ; \operatorname{Im} \Omega>0\} \text { modulo } \operatorname{Sp}_{4}(\mathbb{Z})-\text { action } \\
& =\mathrm{PGL}_{2}(\mathbb{C}) \backslash\{\text { degree six polynomials with simple roots }\} / \mathcal{S}_{6} \\
& =\left\{\left(\xi_{1}, \xi_{2}, \xi_{3}\right) \in(\mathbb{C}-\{0,1\})^{3} ; \xi_{i} \neq \xi_{j} \text { if } i \neq j\right\} \text { modulo } \mathcal{S}_{6}^{\prime} \text {-action }
\end{aligned}
$$

where $\mathcal{S}_{6}$-elements reorder the roots of $P_{6}(z)$, the elements of $\mathrm{PGL}_{2}(\mathbb{C})$ normalize the roots in the form $\left(0,1, \infty, \xi_{1}, \xi_{2}, \xi_{3}\right)$ and then we forget 0,1 and $\infty$ to obtain a $S_{6}^{\prime}$-action on the triples $\left(\xi_{1}, \xi_{2}, \xi_{3}\right)$. In general, there exist $720 \mathcal{S}_{6}^{\prime}$ equivalent such triples. Each of them brings the curve $R(\Omega)$ in a Rosenhain normal form, say

$$
\begin{gathered}
w^{2}=z(z-1)\left(z-\xi_{1}\right)\left(z-\xi_{2}\right)\left(z-\xi_{3}\right) \\
\xi_{1}:=\frac{\theta\left[\begin{array}{ll}
0 & 0 \\
\frac{1}{2} & 0
\end{array}\right]^{2} \theta\left[\begin{array}{ll}
0 & 0 \\
0 & 0
\end{array}\right]^{2}}{\theta\left[\begin{array}{ll}
0 & \frac{1}{2} \\
\frac{1}{2} & 0
\end{array}\right]^{2} \theta\left[\begin{array}{ll}
0 & \frac{1}{2} \\
0 & 0
\end{array}\right]^{2}}, \quad \xi_{2}:=\frac{\theta\left[\begin{array}{ll}
0 & 0 \\
\frac{1}{2} & 0
\end{array}\right]^{2} \theta\left[\begin{array}{ll}
\frac{1}{2} & 0 \\
0 & 0
\end{array}\right]^{2}}{\theta\left[\begin{array}{ll}
0 & \frac{1}{2} \\
\frac{1}{2} & 0
\end{array}\right]^{2} \theta\left[\begin{array}{ll}
\frac{1}{2} & \frac{1}{2} \\
0 & 0
\end{array}\right]^{2}}, \quad \xi_{3}:=\frac{\theta\left[\begin{array}{cc}
\frac{1}{2} & 0 \\
0 & 0
\end{array}\right]^{2} \theta\left[\begin{array}{ll}
0 & 0 \\
0 & 0
\end{array}\right]^{2}}{\theta\left[\begin{array}{ll}
\frac{1}{2} & \frac{1}{2} \\
0 & 0
\end{array}\right]^{2} \theta\left[\begin{array}{ll}
0 & \frac{1}{2} \\
0 & 0
\end{array}\right]^{2}}
\end{gathered}
$$

according to (3) with $z_{i}=\xi_{i}, i=1,2,3$.

\section{Acknowledgements}

The author wishes to thank R.-P. Holzapfel for his clarifying comments during the preparation of the paper. This research is partially supported by a grant $\mathrm{SU}$ 109/2008.

\section{References}

[1] Abel N., Beweis der Unmöglichkeit algebraische Gleichungen von höheren Graden als dem vierten allgemein aufzulösen, J. Reine Angew. Math. 1 (1826) 65-84. 
[2] Artin E., Theorie der Zöpfe, Abhandlungen Hamburg 4 (1926) 47-62; Theory of Braids, Ann. Math. 48 (1947) 101-126.

[3] Behr H., Eine endliche Präsentation der symplektischen Gruppe $\operatorname{Sp}_{4}(\mathbb{Z})$, Math. Z. 141 (1975) 47-56.

[4] Belokolos E. and Enol'skii V., Solutions of Algebraically Integrable Nonlinear Equations and Theta-constants, preprint IMP.9.86, Inst. for Metal Phys., Kiev, 1986.

[5] Coxeter H. and Moser W., Generators and Relations for Discrete Groups, Springer, Berlin, 1972.

[6] Galois E., Ecrits et mémoires mathématiques, Gautier-Villars, Paris, 1962.

[7] Griffiths P. and Harris J., Principles of Algebraic Geometry, Willey, N.Y., 1978.

[8] Guàrdia J., Jacobian Nullwerte and Algebraic Equations, Journal of Algebra 253 (2002) 112-132.

[9] Hermite Ch., Sur la résolution de l'équation du cinquième degré, C. R. Acad. Sci. 46 (1858) 508-515.

[10] Igusa J., Theta Functions, Grund. Math. Wiss. 194, Springer, Berlin, 1972.

[11] Jordan C., Traité des substitution et des équation algébrique, GautierVillars, Paris, 1870.

[12] Klein F., Gleichungen vom siebenten und achten Grade, Math. Ann. 15 (1879) 251-282.

[13] Kronecker L., Sur la résolution de l'équation du cinquième degré, C. R. Acad. Sci. 46 (1858) 1150-1152.

[14] Mumford D., Tata Lectures on Theta II, Birkhäuser, Boston, 1984.

[15] O’Meara O., Lectures on Symplectic Groups, Univ. Notre Dame, Paris, 1976.

[16] Rosenhain G., Abhandlung über die Functionen zweiter Variabler mit vier Perioden, Mem. des savants étrangers 11 (1851) 362-468.

[17] Thomae J., Beitrag zur Bestimmung von $\theta(0,0, \ldots, 0)$ durch die Klassenmoduln algebraischer Functionen, J. Reine Angew. Math. 71 (1870) 201222.

[18] Weber H., Lehrbuch der Algebra, Chelsea, New York, 1898.

Angel Zhivkov

Department of Mathematics \& Informatics

Kliment Ohridski University

Sofia 1164

BULGARIA

E-mail address: zhivkovefmi.uni-sofia.bg 